\title{
SURFACE INTEGRALS APPROACH TO SOLUTION OF SOME FREE BOUNDARY PROBLEMS - II *
}

\author{
Igor Malyshev \\ Department of Mathematics and Computer Science \\ San Jose State University, San Jose, CA 95192
}

\begin{abstract}
This paper is a continuation of the publication [1] where integral equation techniques were applied to the solution of a generalized Stefan problem. The regularization of the corresponding system of nonlinear integral Volterra equations offered here is quite different from that in [1], hence - several new algorithms and numerical experiments. For consistency and easy reference we start this paper with sec.6.
\end{abstract}

Key words: heat equation, free boundary, generalized Stefan problem, system of nonlinear integral equation, singularity, regularization, numerical simulations.

AMS subject classifications: $35 \mathrm{R} 30,35 \mathrm{R} 35$

\section{REGULARIZATION - II}

Let us start immediately from the main system of integral equations (1.6), (1.9), derived in [1], with $\mathrm{K}(\mathrm{x}, \mathrm{t}, \tau, \mathrm{p})$ given by (1.10):

(6.1) $p(t)=r(t)-\frac{1}{\sqrt{\pi}} \int_{0}^{t} p(\tau)\left[\frac{s(t)-s(\tau)}{2(t-\tau)^{3 / 2}}-\frac{p(\tau)}{\sqrt{t-\tau}}\right] e^{-\frac{(s(t)-s(\tau))^{2}}{4(t-\tau)}} d \tau$

* Received: April, 1989. Revised: May, 1989. 


$$
\begin{aligned}
& +\frac{1}{\sqrt{\pi}} \int_{0}^{t} \mu(\tau) \frac{1}{\sqrt{t-\tau}} e^{-\frac{(s(t)-s(\tau))^{2}}{4(t-\tau)}} d \tau, \\
& \mu(t)=h(t)-\int_{0}^{t} p(\tau) K(0, t, \tau, p) d \tau \\
& -\frac{1}{2 \sqrt{\pi}} \int_{0}^{t} \mu(\tau) \frac{s(t)-s(\tau)}{(t-\tau)^{3 / 2}} e^{-\frac{(s(t)-s(\tau))^{2}}{4(t-\tau)}} d \tau .
\end{aligned}
$$

As we have mentioned in [1] some integrals in (6.2) are convergent only conditionally which makes the numerical solution of the system quite "uncomfortable". Before any numerical scheme is applied, this system will be regularized as follows. First, we "remodel" equation (6.2) obtained from the condition (1.7), [1]. Since $\mathrm{W}_{\mathrm{x}}(\mathrm{x}, \mathrm{t}, \mathrm{p})$ can be represented in the form

$$
2 \frac{\partial W(x, t, p)}{\partial x}=\int_{0}^{t} p(\tau) K(x, t, \tau, p) d \tau
$$

we then observe that for $x>0$

$$
\begin{aligned}
& \int_{\tau}^{t} K(x, t, \sigma, p) d \sigma=\frac{\partial}{\partial x} \frac{1}{\sqrt{\pi}} \int_{\tau}^{t}\left[\frac{x+s(t)-s(\sigma)}{2(t-\sigma)^{3 / 2}}\right. \\
& \left.-\frac{p(\sigma)}{\sqrt{t-\sigma}}\right] e^{-\frac{(x+s(t)-s(\sigma))^{2}}{4(t-\sigma)}} d \sigma \\
& =\frac{\partial}{\partial x}\left[\frac{2}{\sqrt{\pi}} \int_{\frac{x+s(t)-s(\tau)}{2 \sqrt{t-\tau}}}^{\infty} e^{-y^{2}} d y\right]=\frac{\partial}{\partial x}\left[1-\operatorname{erf}\left(\frac{x+s(t)-s(\tau)}{2 \sqrt{t-\tau}}\right)\right]
\end{aligned}
$$




$$
=-\frac{1}{\sqrt{\pi(t-\tau)}} e^{-\frac{(x+s(t)-s(\tau))^{2}}{4(t-\tau)}} \equiv T(x, t, \tau, p)
$$

Now, it can be easily verified that

$$
-\frac{\partial T}{\partial \tau}=K(x, t, \tau, p)
$$

Then from (6.3) - (6.5), using integration by parts, we find that

$$
\begin{gathered}
\lim _{x \rightarrow 0^{+}} 2 \frac{\partial W(x, t, p)}{\partial x}=\int_{0}^{t-\Delta} p(\tau) K(0, t, \tau, p) d \tau-\frac{p(t-\Delta)}{\sqrt{\pi \Delta}} e^{-\frac{(s(t)-s(t-\Delta))^{2}}{4 \Delta}} \\
-\int_{t-\Delta}^{t} p^{\prime}(\tau) \frac{1}{\sqrt{\pi(t-\tau)}} e^{-\frac{(s(t)-s(\tau))^{2}}{4(t-\tau)}} d \tau
\end{gathered}
$$

for $\mathrm{t}>0$ and any fixed $0<\Delta \leq \mathrm{t}$, which implies another form of the equation (6.2):

$$
\begin{gathered}
\mu(t)=h(t)-\int_{0}^{t-\Delta} p(\tau) K(0, t, \tau, p) d \tau \\
-\frac{1}{2 \sqrt{\pi}} \int_{0}^{t} \mu(\tau) \frac{s(t)-s(\tau)}{(t-\tau)^{3 / 2}} e^{-\frac{(s(t)-s(\tau))^{2}}{4(t-\tau)} d \tau} d e^{-\frac{(s(t)-s(\tau))^{2}}{4(t-\tau)}} d \tau
\end{gathered}
$$

The equation (6.1) actually does not require any changes and its analysis remains 
exactly the same as in [1]. Nevertheless, for the discussion that follows we represent (6.1) in a regularized form as well:

$$
\begin{aligned}
& p(t)=r(t)-\frac{1}{\sqrt{\pi}} \int_{0}^{t-\Delta} p(\tau)\left[\frac{s(t)-s(\tau)}{2(t-\tau)^{3 / 2}}-\frac{p(\tau)}{\sqrt{t-\tau}}\right] e^{-\frac{(s(t)-s(\tau))^{2}}{4(t-\tau)}} d \tau \\
& +\frac{1}{\sqrt{\pi}} \int_{0}^{t} \mu(\tau) \frac{1}{\sqrt{t-\tau}} e^{-\frac{(s(t)-s(\tau))^{2}}{4(t-\tau)}} d \tau+p(t-\Delta) \operatorname{erf}\left(\frac{s(t)-s(t-\Delta)}{2 \sqrt{\Delta}}\right) \\
& \quad+\int_{t-\Delta}^{t} p^{\prime}(\tau) \operatorname{erf}\left(\frac{s(t)-s(\tau)}{2 \sqrt{t-\tau}}\right) d \tau
\end{aligned}
$$

It is obvious that for $\Delta \rightarrow 0^{+}$(6.7) will reduce to its original form (6.1).

As in [1], after the introduction of

$$
k(t, \tau, p)=\frac{1}{t-\tau} \int_{\tau}^{t} p(z) d z=\frac{s(t)-s(\tau)}{t-\tau}
$$

and

$$
\mathrm{k}(\mathrm{t}, \mathrm{t}-\Delta, \mathrm{p})=\frac{1}{\Delta} \int_{\mathrm{t}-\Delta}^{\mathrm{t}} \mathrm{p}(\mathrm{z}) \mathrm{dz}=\frac{\mathrm{s}(\mathrm{t})-\mathrm{s}(\mathrm{t}-\Delta)}{\Delta}
$$

the system (6.7), (6.6) can be rewritten in the form:

$$
\text { (6.8) } \begin{aligned}
\mathrm{p}(\mathrm{t}) & =\mathrm{r}(\mathrm{t})-\frac{1}{2 \sqrt{\pi}} \int_{0}^{\mathrm{t}-\Delta} \frac{\mathrm{p}(\tau)}{\sqrt{\mathrm{t}-\tau}}[\mathrm{k}(\mathrm{t}, \tau, \mathrm{p})-2 \mathrm{p}(\tau)] \mathrm{e}^{-\frac{1}{4}(\mathrm{t}-\tau) \mathrm{k}^{2}(\mathrm{t}, \tau, \mathrm{p})} \mathrm{d} \tau \\
& +\mathrm{p}(\mathrm{t}-\Delta) \operatorname{erf}\left(\frac{\sqrt{\Delta}}{2} \mathrm{k}(\mathrm{t}, \mathrm{t}-\Delta, \mathrm{p})\right)+\int_{\mathrm{t}-\Delta}^{\mathrm{t}} \mathrm{p}^{\prime}(\tau) \operatorname{erf}\left(\frac{\mathrm{s}(\mathrm{t})-\mathrm{s}(\tau)}{2 \sqrt{\mathrm{t}-\tau}}\right) \mathrm{d} \tau
\end{aligned}
$$




$$
\begin{gathered}
\quad+\frac{1}{\sqrt{\pi}} \int_{0}^{t} \mu(\tau) \frac{1}{\sqrt{t-\tau}} e^{-\frac{1}{4}(t-\tau) k^{2}(t, \tau, p)} d \tau \\
\text { (6.9) } \mu(t)=h(t)-\frac{1}{2 \sqrt{\pi}} \int_{0}^{t} \frac{\mu(\tau)}{\sqrt{t-\tau}} k(t, \tau, p) e^{-\frac{1}{4}(t-\tau) k^{2}(t, \tau, p)} d \tau \\
-\int_{0}^{t-\Delta} \frac{p(\tau)}{4 \sqrt{\pi(t-\tau)}}\left[\frac{2}{t-\tau}-k^{2}(t, \tau, p)+p(\tau) k(t, \tau, p)\right] e^{-\frac{1}{4}(t-\tau) k^{2}(t, \tau, p)} d \tau \\
+\frac{p(t-\Delta)}{\sqrt{\pi \Delta}} e^{-\frac{1}{4} \Delta k^{2}(t, t-\Delta, p)}+\int_{t-\Delta}^{t} \frac{p^{\prime}(\tau)}{\sqrt{\pi(t-\tau)}} e^{-\frac{1}{4}(t-\tau) k^{2}(t, \tau, p)} d \tau .
\end{gathered}
$$

\section{EULER'S METHOD}

Formulas (6.8)-(6.9) open several possibilities. First, we presume that $\mathrm{p}^{\prime}(\tau) \equiv$ 0 in a small interval $\tau \in(\mathrm{t}-\Delta, \mathrm{t})$ (see the last integral in (6.9)), which implies that we can put $p(\tau) \equiv p(t-\Delta)$ for this interval and find, as a result, that

$$
\mathrm{k}(\mathrm{t}, \tau, \mathrm{p})=\mathrm{k}(\mathrm{t}, \mathrm{t}-\Delta, \mathrm{p})=\mathrm{p}(\mathrm{t}-\Delta) \text { for } \tau \in(\mathrm{t}-\Delta, \mathrm{t})
$$

and

$$
\mathrm{k}(\mathrm{t}, \tau, \mathrm{p})=\frac{\mathrm{t}-\Delta-\tau}{\mathrm{t}-\tau} \mathrm{k}(\mathrm{t}-\Delta, \tau, \mathrm{p})+\frac{\Delta}{\mathrm{t}-\tau} \mathrm{p}(\mathrm{t}-\Delta) \text { for } \tau \in(0, \mathrm{t}-\Delta)
$$

The second simplification of the system is based on a product integration technique applied to integrals containing function $\mu(\tau)$ in both equations. Namely, we put $\mu(\tau) \equiv \mu(t-\Delta)$ for all $\tau \in(t-\Delta, t)$ and then find 


$$
\begin{aligned}
& \frac{1}{\sqrt{\pi}} \int_{t-\Delta}^{t} \mu(\tau) \frac{1}{\sqrt{t-\tau}} e^{-\frac{1}{4}(t-\tau) k^{2}(t, \tau, p)} d \tau=\frac{2 \mu(t-\Delta)}{p(t-\Delta)} \operatorname{erf}\left(\frac{1}{2} p(t-\Delta) \sqrt{\Delta}\right), \\
& \frac{1}{2 \sqrt{\pi}} \int_{t-\Delta}^{t} \frac{\mu(\tau)}{\sqrt{t-\tau}} k(t, \tau, p) e^{-\frac{1}{4}(t-\tau) k^{2}(t, \tau, p)} d \tau=\mu(t-\Delta) \operatorname{erf}\left(\frac{1}{2} p(t-\Delta) \sqrt{\Delta}\right) .
\end{aligned}
$$

This technique is equivalent to the piecewise constant approximation of the integrands $p(t)$ and $\mu(t)$.

Now, starting with $p\left(t_{0}\right)=p(0)=0$ and $\mu\left(t_{0}\right)=\mu(0)=0$, all successive values of $p\left(t_{k}\right)$ and $\mu\left(t_{k}\right)$ (with the $\left.\Delta=t_{k}-t_{k-1}\right)$ can be computed directly from the system:

$$
\begin{gathered}
\mathrm{p}\left(\mathrm{t}_{\mathrm{k}}\right)=\mathrm{r}\left(\mathrm{t}_{\mathrm{k}}\right)+\frac{1}{\sqrt{\pi}} \int_{0}^{\mathrm{t} k-1} \frac{\mu(\tau)}{\sqrt{\mathrm{t}_{\mathrm{k}}-\tau}} \mathrm{e}^{-\frac{1}{4}\left(\mathrm{t}_{\mathrm{k}}-\tau\right) \mathrm{k}^{2}\left(\mathrm{t}_{\mathrm{k}}, \tau, \mathrm{p}\right)} \mathrm{d} \tau \\
-\frac{1}{2 \sqrt{\pi}} \int_{0}^{t_{\mathrm{k}-1}} \frac{\mathrm{p}(\tau)}{\sqrt{\mathrm{t}_{\mathrm{k}}-\tau}}\left[\mathrm{k}\left(\mathrm{t}_{\mathrm{k}}, \tau, \mathrm{p}\right)-2 \mathrm{p}(\tau)\right] \mathrm{e}^{-\frac{1}{4}\left(\mathrm{t}_{\mathrm{k}}-\tau\right)_{\mathrm{k}}^{2}\left(\mathrm{t}_{\mathrm{k}}, \tau, \mathrm{p}\right)} \mathrm{d} \tau \\
+\left[\mathrm{p}\left(\mathrm{t}_{\mathrm{k}-1}\right)+\frac{2 \mu\left(\mathrm{t}_{\mathrm{k}-1}\right)}{\mathrm{p}\left(\mathrm{t}_{\mathrm{k}-1}\right)}\right] \operatorname{erf}\left(\frac{\sqrt{\Delta}}{2} \mathrm{p}\left(\mathrm{t}_{\mathrm{k}-1}\right)\right),
\end{gathered}
$$

$$
\mu\left(t_{k}\right)=h\left(t_{k}\right)-\frac{1}{2 \sqrt{\pi}} \int_{0}^{t} \frac{\mu(\tau)}{\sqrt{t_{k}-\tau}} k\left(t_{k}, \tau, p\right) e^{-\frac{1}{4}\left(t_{k}-\tau\right) k^{2}\left(t_{k}, \tau, p\right)} d \tau
$$

$$
-\int_{0}^{\mathrm{t} k-1} \frac{\mathrm{p}(\tau)}{4 \sqrt{\pi\left(\mathrm{t}_{\mathrm{k}}-\tau\right)}}\left[\frac{2}{\mathrm{t}_{\mathrm{k}}-\tau}-\mathrm{k}^{2}\left(\mathrm{t}_{\mathrm{k}}, \tau, \mathrm{p}\right)+\mathrm{p}(\tau) \mathrm{k}\left(\mathrm{t}_{\mathrm{k}}, \tau, \mathrm{p}\right)\right] \mathrm{e}^{-\frac{1}{4}\left(\mathrm{t}_{\mathrm{k}}-\tau\right) \mathrm{k}^{2}\left(\mathrm{t}_{\mathrm{k}}, \tau, \mathrm{p}\right)} \mathrm{d} \tau
$$




$$
+\frac{p\left(t_{k-1}\right)}{\sqrt{\pi \Delta}} e^{-\frac{1}{4} \Delta p^{2}\left(t_{k-1}\right)}-\mu\left(t_{k-1}\right) \operatorname{erf}\left(\frac{1}{2} p\left(t_{k-1}\right) \sqrt{\Delta}\right)
$$

where function $k\left(t_{k}, \tau, p\right)$ is computed from the recursion formula (7.1).

\section{OTHER POSSIBLE SCHEMES}

Considering the system (6.8)-(6.9) again, we can try to solve it by the Picard iterations method, Euler's method or a mixture of both. For example, if we do not restrict $\mathrm{p}^{\prime}(\mathrm{t})$ as in section 7 of this paper, we find (using notation accepted in $(7.2)-(7.3))$

$$
\mathrm{p}^{\prime}(\tau)=\frac{1}{\Delta}\left(\mathrm{p}\left(\mathrm{t}_{\mathrm{k}}\right)-\mathrm{p}\left(\mathrm{t}_{\mathrm{k}-1}\right)\right) \text { for all } \tau \in\left(\mathrm{t}_{\mathrm{k}-1}, \mathrm{t}_{\mathrm{k}}\right) \text {; }
$$

which immediately implies:

$$
\begin{gathered}
k\left(t_{k}, t_{k-1}, p\right)=\alpha_{1} p\left(t_{k}\right)+\alpha_{2} p\left(t_{k-1}\right), \\
k\left(t_{k}, \tau, p\right)=\frac{t_{k-1}-\tau}{t_{k}-\tau} k\left(t_{k-1}, \tau, p\right)+\frac{\Delta}{t_{k}-\tau} k\left(t_{k}, t_{k-1}, p\right) \text { for } \tau \in\left(0, t_{k-1}\right), \\
k\left(t_{k}, \tau, p\right)=\beta_{1} p\left(t_{k}\right)+\beta_{2} p\left(t_{k-1}\right) \text { for } \tau \in\left(t_{k-1}, t_{k}\right),
\end{gathered}
$$$$
\left(\alpha_{1}, \alpha_{2}, \beta_{1}, \beta_{2} \geq 0, \alpha_{1}+\alpha_{2}=1, \beta_{1}+\beta_{2}=1 \text {; the case of } \alpha_{1}=\alpha_{2}=\beta_{1}=\beta_{2}=1 / 2\right.
$$

may do perfectly well, with any other choice depending on circumstances).

Now, if we employ a mixture of two methods, i.e. the method of successive approximations to find $\mathrm{p}\left(\mathrm{t}_{\mathrm{k}}\right)$ and Euler's method for $\mu\left(\mathrm{t}_{\mathrm{k}}\right)$, then for the $n$-th approximation of $\mathrm{p}\left(\mathrm{t}_{\mathrm{k}}\right)$ we get: 


$$
p^{(n)}\left(t_{k}\right)=r\left(t_{k}\right)+p\left(t_{k-1}\right) \operatorname{erf}\left(\frac{\sqrt{\Delta}}{2} k_{n-1}\left(t_{k}, t_{k-1}, p\right)\right)
$$$$
-\frac{1}{2 \sqrt{\pi}} \int_{0}^{t_{k}-1} \frac{p(\tau)}{\sqrt{t_{k}-\tau}}\left[k_{n-1}\left(t_{k}, \tau, p\right)-2 p(\tau)\right] e^{-\frac{1}{4}\left(t_{k}-\tau\right) k_{n-1}^{2}\left(t_{k}, \tau, p\right)} d \tau
$$

$$
+\int_{t_{k-1}}^{t_{k}} \frac{1}{\Delta}\left[p^{(n-1)}\left(t_{k}\right)-p\left(t_{k-1}\right)\right] \operatorname{erf}\left(\frac{\sqrt{\Delta}}{2} k_{n-1}\left(t_{k}, \tau, p\right)\right) d \tau
$$

$$
+\frac{1}{\sqrt{\pi}} \int_{0}^{t_{k-1}} \frac{\mu(\tau)}{\sqrt{t_{k}-\tau}} e^{-\frac{1}{4}\left(t_{k}-\tau\right) k_{n-1}^{2}\left(t_{k}, \tau, p\right)} d \tau+\frac{2 \mu\left(t_{k-1}\right)}{p\left(t_{k-1}\right)} \operatorname{erf}\left(\frac{\sqrt{\Delta}}{2} k_{n-1}\left(t_{k}, t_{k-1}, p\right)\right),
$$

where

$$
\begin{gathered}
k_{n-1}\left(t_{k}, t_{k-1}, p\right)=\alpha_{1} p^{(n-1)}\left(t_{k}\right)+\alpha_{2} p\left(t_{k-1}\right) \\
k_{n-1}\left(t_{k}, \tau, p\right)=\frac{t_{k-1}-\tau}{t_{k}-\tau} k\left(t_{k-1}, \tau, p\right)+\frac{\Delta}{t_{k}-\tau} k_{n-1}\left(t_{k}, t_{k-1}, p\right) \text { for } \tau \in\left(0, t_{k-1}\right), \\
k_{n-1}\left(t_{k}, \tau, p\right)=\beta_{1} p^{(n-1)}\left(t_{k}\right)+\beta_{2} p\left(t_{k-1}\right) \text { for } \tau \in\left(t_{k-1}, t_{k}\right)
\end{gathered}
$$

The expression for $\mu\left(t_{k}\right)$ is similar to that in (7.3). We use it after $p\left(t_{k}\right) \equiv$ $\mathrm{p}^{(\mathrm{n})}\left(\mathrm{t}_{\mathrm{k}}\right)$ and $\mathrm{k}\left(\mathrm{t}_{\mathrm{k}}, \mathrm{t}, \mathrm{p}\right) \equiv \mathrm{k}_{\mathrm{n}}\left(\mathrm{t}_{\mathrm{k}}, \mathrm{t}, \mathrm{p}\right)$ are found from (8.1):

$$
\mu\left(t_{k}\right)=h\left(t_{k}\right)-\mu\left(t_{k-1}\right) \operatorname{erf}\left(\frac{\sqrt{\Delta}}{2} k\left(t_{k}, t_{k-1}, p\right)\right)
$$




$$
\begin{aligned}
& -\int_{0}^{t_{k}-1} \frac{p(\tau)}{4 \sqrt{\pi\left(t_{k}-\tau\right)}}\left[\frac{2}{t_{k}-\tau}-k^{2}\left(t_{k}, \tau, p\right)+p(\tau) k\left(t_{k}, \tau, p\right)\right] e^{-\frac{1}{4}\left(t_{k}-\tau\right) k^{2}\left(t_{k}, \tau, p\right)} d \tau \\
& +\frac{p\left(t_{k-1}\right)}{\sqrt{\pi \Delta}} e^{-\frac{1}{4} \Delta k^{2}\left(t_{k}, t_{k-1}, p\right)}+\int_{t_{k}-1}^{t_{k}} \frac{p\left(t_{k}\right)-p\left(t_{k-1}\right)}{\sqrt{\pi \Delta\left(t_{k}-\tau\right)}} e^{-\frac{1}{4}\left(t_{k}-\tau\right) k^{2}\left(t_{k}, \tau, p\right)} d \tau \\
& -\frac{1}{2 \sqrt{\pi}} \int_{0}^{t_{k-1}} \frac{\mu(\tau)}{\sqrt{t_{k}-\tau}} k\left(t_{k}, \tau, p\right) e^{-\frac{1}{4}\left(t_{k}-\tau\right) k^{2}\left(t_{k}, \tau, p\right)} d \tau .
\end{aligned}
$$

Obviously, if in addition we drop restrictions on $\mu$ (see section 7, where $\mu(\tau)$ $\equiv \mu\left(t_{k-1}\right)$ for $\left.\tau \in\left(t_{k-1}, t_{k}\right)\right)$, we shall get a system where the Picard approximation technique has to be applied to both equations.

\section{ON NUMERICAL TECHNIQUES AND SIMULATIONS}

The regularized equations (8.1)-(8.2) (or their modifications as discussed above) show that we have to deal with an integrable type of singularity only. Any standard numerical integration scheme in combination with the Picard approximation technique can be applied now to realize the algorithms in sections 7 and 8 . The priority should be given to those numerically stable approximation methods that guarantee small accumulated discretization error in case of a nonlinear integral Volterra equation with weak singularity.

After finding the moving boundary $s(t)$ (and the density function $\mu$ ) from one of the systems above, we obtain the solution $\mathrm{u}(\mathrm{x}, \mathrm{t})$ of the problem in the form (1.5). On the other hand, the knowledge of $s(t)$ allows to solve the direct boundary value problem (0.1)-(0.3), say, by the finite difference method. Our numerical experiments on verification of the inverse problem method showed good resemblance between (1.5) and the "direct"solution. 
Acknowledgements.

The author thanks Drs. V. Naroditsky and H. Morris for valuable discussions on the problems touched upon in this paper and B. Bekins for conducting numerical experiments.

\section{REFERENCES}

[1] Malyshev, I., Morris, H., Naroditsky, V., Romanyuk, L., Surface integrals approach to solution of some free boundary problems, $J$. of Applied Mathematics and Simulation, V. 1, No. 4, 305-323, (1988).

[2] Crank, J., Free and Moving Boundary Problems, Oxford University Press, N.Y., (1984).

[3] Press, W. H., Flannery, B. P., Teukolsky, S. A., Vetterling, W. T., Numerical Recipes, Cambridge University Press, N.Y., (1986).

[4] Linz, P., Analytical and Numerical Methods for Volterra Equations, SIAM, (1985). 


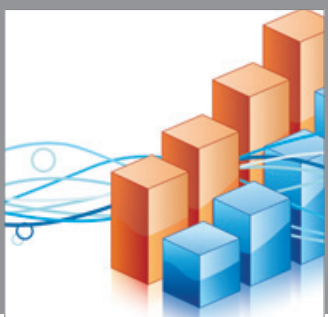

Advances in

Operations Research

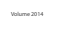

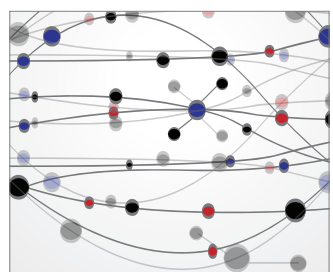

\section{The Scientific} World Journal
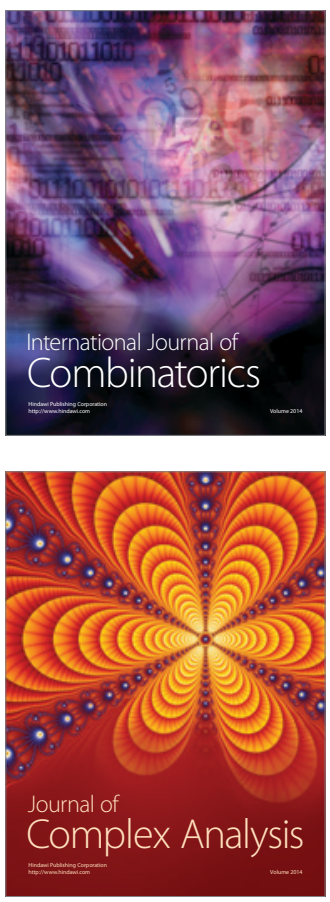

International Journal of

Mathematics and

Mathematical

Sciences
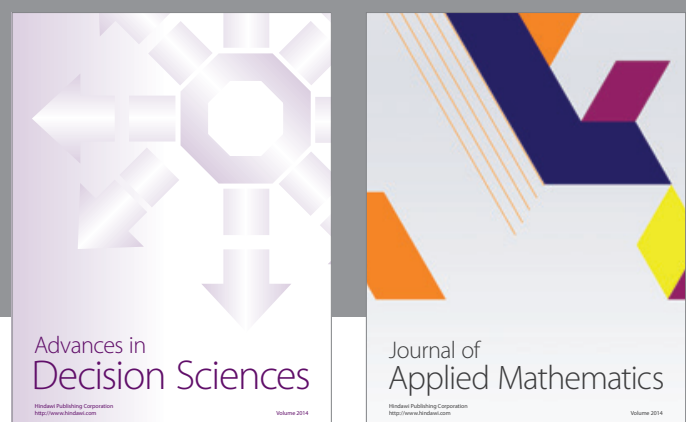

Journal of

Applied Mathematics
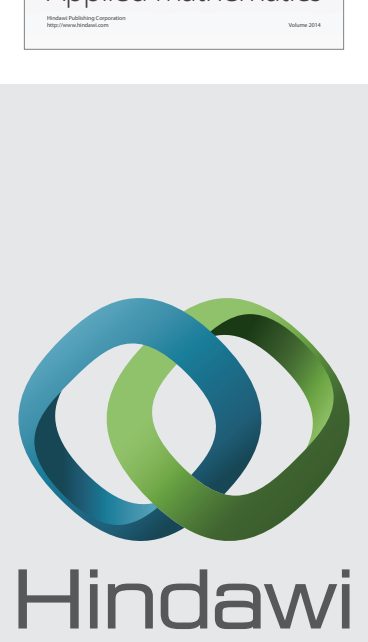

Submit your manuscripts at http://www.hindawi.com
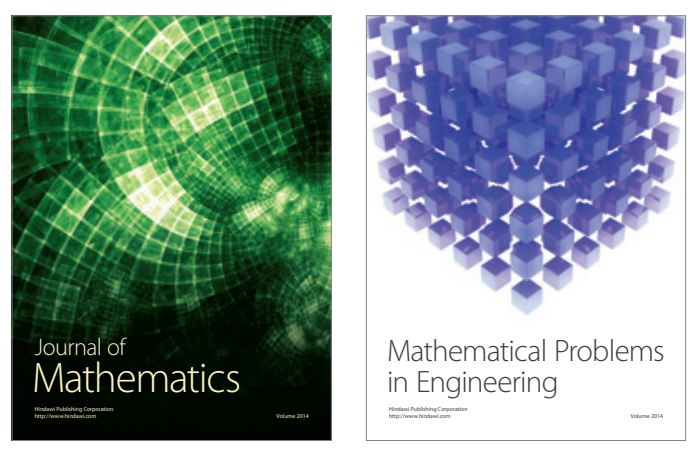

Mathematical Problems in Engineering
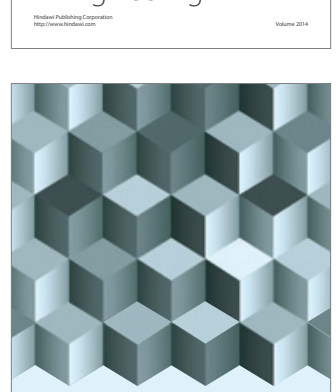

Journal of

Function Spaces
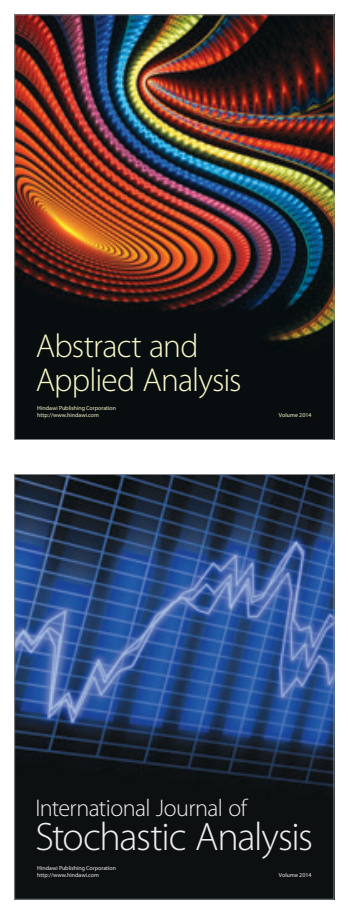

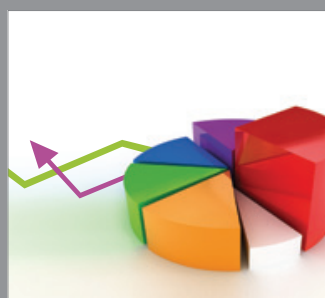

ournal of

Probability and Statistics

Promensencen
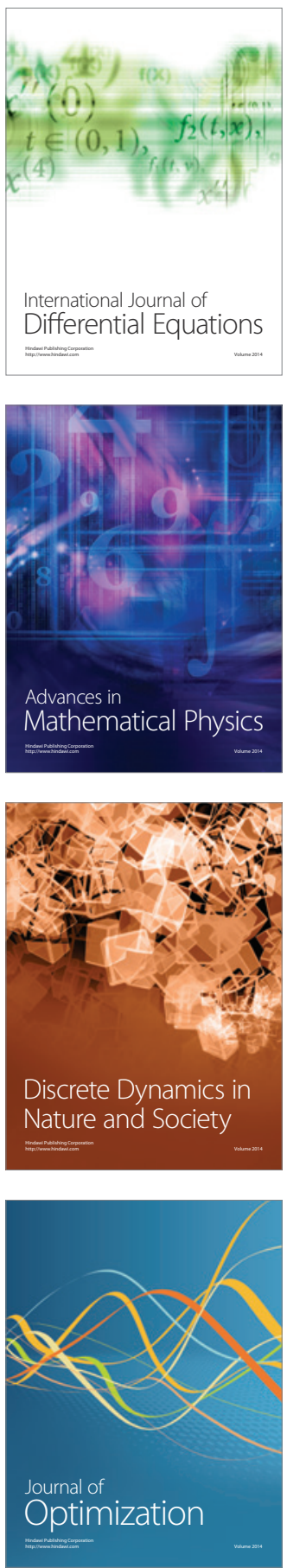